COLO-HEP-475

October 2001

\title{
Simulating dynamical fermions with smeared links
}

\author{
A. Hasenfratz ${ }^{\mathrm{a}}$ and F. Knechtli ${ }^{\mathrm{a} *}$ \\ ${ }^{a}$ Department of Physics, University of Colorado, Campus Box 390, Boulder, CO 80309, USA
}

Smearing the gauge links of dynamical configurations removes small scale unphysical vacuum fluctuations and thus improves the chiral properties of lattice fermions. Recently we proposed the hypercubic smearing (HYP) that improves the flavor symmetry of staggered fermions by an order of magnitude with only minimal distortions at small distances. We describe a new algorithm to simulate dynamical HYP fermions based on the standard pure gauge overrelaxation and heatbath updates. The algorithm has been used to simulate four and two flavors of staggered fermions. Unlike standard dynamical simulation techniques, this algorithm does not loose efficiency at small quark masses.

\section{Introduction}

In this article we study an algorithm for simulating a fermionic system described by the action

$S=S_{g}(U)-\operatorname{tr} \ln \left[Q^{\dagger}(V) Q(V)\right]$,

where $S_{g}(U)$ is the pure gauge action and $Q(V)$ is the fermionic matrix. The gauge connections between fermions are smeared links $V$ which are constructed deterministically from the dynamical thin links $U$. Since each smeared link is a local combination of a finite number of thin links, the system where the fermions couple via smeared links is in the same universality class as the system with thin links.

It has been demonstrated that smeared links improve flavor symmetry with staggered fermions, chiral symmetry with Wilson-type clover fermions and they are useful in the construction of an overlap Dirac operator. The problem is that the standard simulation algorithms for dynamical fermions (Hybrid Monte Carlo (HMC) or R algorithms) involve the computation of the gauge force $\partial Q(V) / \partial U$, which is either very complicated or likely impossible if the smeared links are projected onto $\mathrm{SU}(3)$.

\section{New algorithm}

We propose for the simulation of the system described by eq. (1) a two step updating

1) updating of a set of thin links $\{U\} \rightarrow\left\{U^{\prime}\right\}$ which satisfies detailed balance for $S_{g}(U)$

*new address: Institut für Physik, Humboldt-Universität zu Berlin, Invalidenstr. 110, 10115 Berlin, Germany
2) accept/reject step with acceptance probability

$$
P_{\mathrm{acc}}=\min \left\{1, \frac{\operatorname{det}\left[Q^{\dagger}\left(V^{\prime}\right) Q\left(V^{\prime}\right)\right]}{\operatorname{det}\left[Q^{\dagger}(V) Q(V)\right]}\right\},
$$

where $V^{\prime}$ are the smeared links constructed from the updated thin links

As concerns step 1) our choice for $S_{g}$ is the Wilson plaquette action and the updating is performed either with microcanonical overrelaxation or with Cabibbo-Marinari heatbath. With overrelaxation we update all links within some finite block of the lattice (the sequence of the updates in the block has to be symmetrized), with heatbath the links to be updated are chosen randomly.

In step 2), we use a stochastic estimator to evaluate the ratio of fermionic determinants

$$
\begin{aligned}
P_{\mathrm{acc}}^{\prime} & =\min \{1, \exp \Delta S\} \\
\Delta S & =\xi^{\dagger}\left[Q^{\dagger}\left(V^{\prime}\right) Q\left(V^{\prime}\right)-Q^{\dagger}(V) Q(V)\right] \xi,
\end{aligned}
$$

where the vector $\xi$ is generated according to the probability distribution

$P(\xi) \propto \exp \left\{-\xi^{\dagger} Q^{\dagger}\left(V^{\prime}\right) Q\left(V^{\prime}\right) \xi\right\}$.

\section{The HYP action}

In the following we describe the application of this algorithm to the cases of $n_{f}=4$ and $n_{f}=2$ flavors of staggered fermions coupled via hypercubic (HYP) smeared links. The definition of HYP links and their properties are discussed in [1,2]. In the natural formulation of four flavors of staggered quarks, the continuum SU(4) flavor symmetry is broken to $\mathrm{U}(1)$ at finite lattice spacing. 
The pion spectrum has only one true Goldstone pion $\pi_{\mathrm{G}}$, the other 14 pions remain massive when the quark mass goes to zero. The mass splitting between a non-Goldstone pion $\pi$ and $\pi_{\mathrm{G}}$ can be parametrized by the quantity [3]

$\delta_{2}=\frac{m_{\pi}^{2}-m_{\pi_{\mathrm{G}}}^{2}}{m_{\rho}^{2}-m_{\pi_{\mathrm{G}}}^{2}}$

evaluated at $m_{\pi_{\mathrm{G}}} / m_{\rho}=0.55$. With a flavor symmetric action $\delta_{2}=0$ for all the pions $\pi$. We computed $\delta_{2}$ for the two lightest non-Goldstone pions (for the notation see [1] ) on a set of quenched $8^{3} \times 24$ lattices generated with Wilson pure gauge action at $\beta=5.7(a=0.17 \mathrm{fm})$, using three different valence quark actions: standard staggered action with thin and HYP smeared links and improved Asqtad action of the MILC collaboration 渵. The results are shown in table 1 . Flavor sym-

Table 1

Flavor symmetry breaking for different valence quark actions at lattice spacing $a=0.17 \mathrm{fm}$.

\begin{tabular}{|c|c|c|c|}
\hline Action $/ \delta_{2}$ & Thin & Asqtad & HYP \\
\hline \hline$\pi_{i, 5}$ & $0.594(25)$ & $0.191(22)$ & $0.086(14)$ \\
\hline$\pi_{i, j}$ & $0.72(6)$ & $0.32(4)$ & $0.150(24)$ \\
\hline
\end{tabular}

metry violations with the HYP action are reduced by an order of magnitude with respect to the thin link action and by a factor of two with respect to the Asqtad action.

\section{4. $n_{f}=4$ HYP staggered fermions}

We consider the matrix $Q$ in eq. (11) given by

$$
\begin{aligned}
Q_{i, j} & =M_{i, j} \equiv 2(a m) \delta_{i, j}+D_{i, j}(V), \quad(7) \\
D_{i, j}(V) & =\sum_{\mu} \eta_{i, \mu}\left(V_{i, \mu} \delta_{i, j-\hat{\mu}}-V_{i-\hat{\mu}, \mu}^{\dagger} \delta_{i, j+\hat{\mu}}\right),
\end{aligned}
$$

which describes four flavors of staggered fermions coupled via HYP smeared links $V$. In the accept/reject step we rewrite the fermion matrix as

$Q(V)=Q_{r}(V) A(V)$,

$A(V)=\exp \left[\alpha_{4} D^{4}(V)+\alpha_{2} D^{2}(V)\right]$.

In this way we achieve that an effective action

$S_{\text {eff }}=-2 \alpha_{4} \operatorname{RetrD}{ }^{4}-2 \alpha_{2} \operatorname{Re} \operatorname{trD}^{2}$ is removed from the fermion determinant. The acceptance probability in eq. (28) becomes $\tilde{P}_{\text {acc }}=$ $\min \left\{1, \exp \left[S_{\text {eff }}(V)-S_{\text {eff }}\left(V^{\prime}\right)\right] \exp \Delta S_{r}\right\}$,

where the stochastic part $\Delta S_{r}$ is computed like in eqs. (因河) with $Q_{r}$ instead of $Q$. The real parameters $\alpha_{4}$ and $\alpha_{2}$ are optimized to maximize $\tilde{P}_{\text {acc }}$ and we use $\alpha_{4}=-0.006, \alpha_{2}=-0.18$ [5]. The point we would like to emphasize is that this algorithm is not effective with thin links. Smeared links constrain the fluctuations of the stochastic estimator $\exp \Delta S_{r}$ and make the algorithm efficient.

In Monte Carlo simulations we separate the measurements of the observables by $N_{\mathrm{OR}}$ overrelaxation and $N_{\mathrm{HB}}$ heatbath two-step updatings, changing $t_{\mathrm{OR}}$ and $t_{\mathrm{HB}}$ links respectively. At a lattice spacing $a \sim 0.17 \mathrm{fm}$ and correlation length $m_{\pi_{\mathrm{G}}} r_{0}=2.0$ we simulated the HYP algorithm on a $8^{3} \times 24$ lattice with $\left(t_{\mathrm{OR}}=128, N_{\mathrm{OR}}=160\right)$ and $\left(t_{\mathrm{HB}}=200, N_{\mathrm{HB}}=80\right)$ with an acceptance of $\sim 20 \%$. If we run the standard thin link action with HMC at approximately matched physical parameters, we obtain comparable autocorrelations in the observables when the measurements are separated by a trajectory of unit time length. We can then compare the time costs of the two algorithms which are about 7 times larger for the HYP algorithm.

With the new algorithm the quark mass can be lowered to values impractical with standard fermionic algorithms. A similar test as described above at $m_{\pi_{\mathrm{G}}} r_{0}=1.6$ shows that the HYP algorithm is about 3 times slower than the standard thin link HMC.

A drawback of our algorithm is that as the lattice volume increases the numbers of the updated links $t_{\mathrm{OR}}$ and $t_{\mathrm{HB}}$ have to be kept unchanged, consequently the numbers of updatings $N_{\mathrm{OR}}$ and $N_{\text {HB }}$ have to be scaled with the volume to keep the autocorrelation times unchanged. On the other hand as the continuum limit is approached the numbers of links $t_{\mathrm{OR}}$ and $t_{\mathrm{HB}}$ which can be effectively updated scale: the physical volume of the updated region is constant.

\section{5. $n_{f}=2$ HYP staggered fermions}

We consider eq. (1) with

$Q^{\dagger} Q=\left(M^{\dagger} M\right)^{1 / 2}$, 
where $M$ is the four flavor staggered fermion matrix. Perturbative arguments indicate that eq. (12) is equivalent in the continuum to a non-local theory of two flavors of quarks [6].

To evaluate the square root in eq. (12) we use a polynomial approximation. We write $x^{1 / 2}=$ $x x^{-1 / 2}=x P_{-1 / 2}(x)$ with

$P_{-1 / 2}(x)=\lim _{n \rightarrow \infty} P_{-1 / 2}^{(n)}(x)=\lim _{n \rightarrow \infty} \sum_{i=0}^{n} c_{i}^{(n)} x^{i}$.

The coefficients $c_{i}$ are found by minimizing [7]

$I=\int_{0}^{\lambda} d x\left(x^{-1 / 2}-P_{-1 / 2}^{(n)}(x)\right)^{2} x$.

For $n$ even the roots $r_{i}$ of $P_{-1 / 2}^{(n)}$ come in complex conjugate pairs, we can write

$P_{-1 / 2}^{(n)}(x)=q_{(-1 / 2)}^{(n)}(x) q_{(-1 / 2)}^{(n) \dagger}(x)$,

where $q_{(-1 / 2)}^{(n)}(x)=\sqrt{c_{n}^{(n)}} \prod_{i=1}^{n / 2}\left(x-r_{i}^{(n)}\right)$. This allows us to identify

$Q=\lim _{n \rightarrow \infty} M q_{(-1 / 2)}^{(n)}\left(M^{\dagger} M\right)$.

We note that the spectrum of $M^{\dagger} M=4(a m)^{2}-$ $D^{2}$ is bound from below exactly by $4(a m)^{2}$ and the maximum eigenvalue fluctuates around the free field value $16+4(\mathrm{am})^{2}$. We set $\lambda=1$ in eq. (14) and then rescale the polynomial to use it in the range $x \in(0,18)$.

In the accept/reject step eq. (3) the stochastic estimator $\exp \Delta S$ is evaluated as

$$
\begin{aligned}
\Delta S^{(n)} & =\xi^{\dagger}\left[P_{-1 / 2}^{\prime(n)} M^{\prime \dagger} M^{\prime}-P_{-1 / 2}^{(n)} M^{\dagger} M\right] \xi \\
\xi & =q_{-1 / 2}^{\prime(m) \dagger} R
\end{aligned}
$$

where $M^{\prime} \equiv M\left(V^{\prime}\right)$, etc. and $R$ is a random Gaussian vector. The systematic error due to the finite order $n$ of the polynomial is $\Delta^{(n)}=P_{-1 / 2}^{(n)}-$ $P_{-1 / 2}$. It is possible to improve eq. (17) such that

$$
\left(\Delta S^{(n)}\right)_{\mathrm{imp}}=\Delta S^{(\infty)}+\mathcal{O}\left(\left(\Delta^{(n)}\right)^{2}\right) .
$$

A similar improvement is possible for the vector $\xi$ in eq. (18) but we decided to take a higher order $m=128$ of the polynomial. In the actual computation in order to increase the acceptance probability of the stochastic estimator we split the fermion matrix $M=M_{r} A$ like in the $n_{f}=4$ case, eqs. (8-9).

In the evaluation of the polynomial eq. (15) the ordering of the roots $r_{i}$ is important for the reduction of numerical round-off errors. We emphasize that the smeared links make the polynomial approximation work with lower order polynomials. In order to test the accuracy of the polynomial approximation we looked at the quantities

$\Delta P_{\mathrm{acc}}^{\left(n, n^{\prime}\right)}=P_{\mathrm{acc}}^{(n)}-P_{\mathrm{acc}}^{\left(n^{\prime}\right)}$,

where $P_{\text {acc }}^{(n)}=\min \left\{1, \exp \left(\Delta S^{(n)}\right)_{\mathrm{imp}}\right\}$ is the acceptance probability using $n$th order polynomial, comparing $n=32,64,128$ with $n^{\prime}=256$. We found that $\Delta P_{\text {acc }}=\mathcal{O}\left(10^{-2}\right)$ for $n=32$ and decreases to $\mathcal{O}\left(10^{-4}\right)$ for $n=128$. Moreover $\Delta P_{\text {acc }}$ does not increase significantly as the mass decreases or the lattice volume increases or the lattice spacing decreases. To answer the question of which accuracy is needed, we performed simulations with different values $n=32,64,128$ but all other parameters fixed. First numerical results for the chiral condensate show agreement within the statistical errors for all orders $n$ of the polynomial but much longer runs are needed to identify the systematic errors of a low order polynomial. The properties of the polynomial approximation have been studied extensively in [7] and we plan to follow some of those methods in the future.

\section{REFERENCES}

1. A. Hasenfratz and F. Knechtli, Phys. Rev. D64, 034504 (2001).

2. A. Hasenfratz, R. Hoffmann and F. Knechtli, these proceedings.

3. K. Orginos and D. Toussaint (MILC), Phys. Rev. D59, 014501 (1999).

4. K. Orginos, D. Toussaint and R. L. Sugar (MILC), Phys. Rev. D60, 054503 (1999).

5. F. Knechtli and A. Hasenfratz, Phys. Rev. D63, 114502 (2001).

6. C. Bernard and M. Golterman, Phys. Rev. D49, 486 (1994).

7. I. Montvay, Nucl. Phys. B466, 259 (1996); Comput. Phys. Commun. 109, 144 (1998). 